\title{
Letter
}

\section{Role of static inductance on ion beam emission in plasma focus devices}

\author{
F A RZIN M. A GHAMIR and REZA A. BEHBAHAN I \\ Department of Physics, University of Tehran, N. Kargar Ave, Tehran 143399, Iran \\ (aghamir@ut.ac.ir)
}

(Received 6 October 2011; revised 14 March 2012; accepted 14 March 2012; first published online 1 May 2012)

\begin{abstract}
The role of static inductance on ion beam emission in plasma focus devices, based on anomalous resistivity, is reported. The effect of gas pressure variation on the process of energy transfer into plasma is investigated and discussed by using Lee's model. The dependence of ion beam production on filling gas pressure along with change in static inductance is studied. The results show that increase of static inductance in a specific range of operating pressure can enhance the efficiency of dense plasma focus device.
\end{abstract}

There have been many studies on ion beam production and emission mechanism in plasma focus devices in recent years. Different ion beam emission mechanisms have been proposed such as induced electric field [1-5], anomalous resistive electric field [6], hybrid model, which is an induced electric field model coupled with an anomalous resistive electric field [7], gyro-reflection acceleration model [8], etc. Moreover, many experiments have been performed in view of changes in some of the device parameters such as current sheath velocity, anode tip [9], and gas pressure [4, 10]. Although all of these experiments have tried to clarify the mechanisms of ion beam emission and the effective parameters in plasma focus devices, it is still difficult to understand globally the ion beam production mechanisms along with the role of effective parameters in plasma focus devices.

The main goal of this paper is to study the effect of inductance variation on ion beam emission. Lee's model [11] has been used to describe the phenomena that occur in relation to the variations of gas pressure at a given value of static inductance. Using Lee's analysis, the effect of change in static inductance on ion beam emission has been investigated.

Lee's model which is based on the coupling between plasma and current sheath dynamics, as well as plasma thermodynamics, and radiation processes along with device circuit parameters has been extensively and successfully applied to different plasma focus devices from low- to high-energy machines such as UNU/ICTP PFF [12, 13], NX1 and NX2 [14], Stuttgart DPF [15], and PF1000 [12, 16]. This model assumes that the plasma focus current trace is the fundamental signal from which all of the occurring phenomena can be extracted.

The effect of gas pressure on ion beam emission has been studied in various plasma focus devices. The results have shown that the highest ion beam energy and flux emitted from these devices usually occur in low operating pressure values. It is clear that high energetic ions with high flux intensities are the results of a phenomenon that increases the transfer of energy from plasma to ions. The current trace is the key indicator of energy transfer into plasma because of any phenomenon taking place in a plasma focus device.

According to Lee's model, the total amount of energy transferred into plasma (TETIP), which is a part of the energy stored in magnetic field, can be expressed as

$$
\text { TETIP }=\int R_{\mathrm{d}} I_{\mathrm{p}}^{2} d t+\int R_{\mathrm{p}} I_{\mathrm{p}}^{2} d t
$$

where

$$
R_{\mathrm{d}}=0.5\left(d L_{\mathrm{p}} / d t\right)
$$

is the dynamic resistance [17] in which $L_{\mathrm{p}}$ is the plasma inductance, $I_{\mathrm{p}}$ is the plasma current, and $R_{\mathrm{p}}$ is the plasma resistance, which can be expressed as

$$
R_{\mathrm{p}}=l /\left(\pi r_{\mathrm{p}}^{2} \sigma\right) .
$$

Here $l, r_{\mathrm{p}}$, and $\sigma$ are the length of the compressed plasma column, plasma radius, and plasma electrical conductivity, respectively.

In order to obtain the required information from current trace signal, different current fitting processes were carried out at different values of pressure in accordance with Lee's computational model [17]. These fittings showed good agreement between the measured current traces and the computed ones for the device operating at high-pressure range (0.8-2 mbar). As Fig. 1 shows, the fitting process for low-pressure values, within the range of $0.1-0.6 \mathrm{mbar}$, resulted in the separation of the computed current trace from the measured one. This was observed for all values of this pressure range (typical fitting parameters for argon gas at a charging voltage of $18 \mathrm{kV}$ were the static inductance $L_{0}=156 \mathrm{nH}$, the 


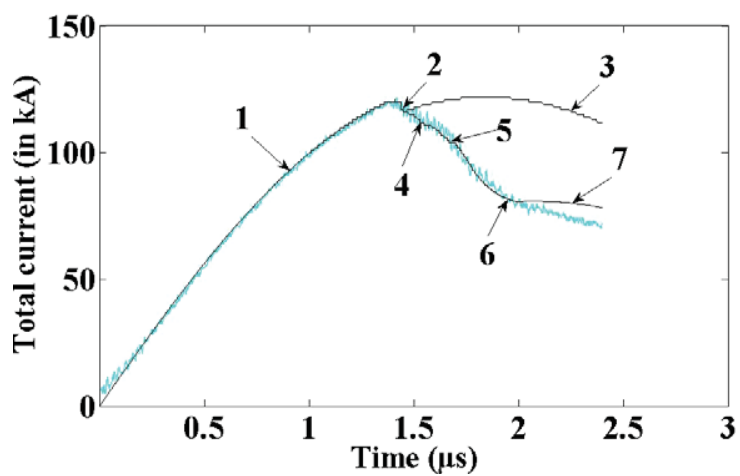

Figure 1. (Colour online) Measured (dark color dotted line) vs. computed (light color smooth lines) current traces. 1 points to the common parts of the measured, 1st, and 2 nd computed current traces. 2 to 7 point to the end of the regular dip, the last part of the 1st computed trace (first fitting), the end of $R_{1}$, the end of $R_{2}$, the end of $R_{3}$, and the last part of 2 nd computed trace (second fitting), respectively.

capacitance $C_{0}=10 \mu \mathrm{F}$, the cathode radius, $b=3 \mathrm{~cm}$, the anode radius $a=1 \mathrm{~cm}$, the anode length $Z_{0}=11 \mathrm{~cm}$, the stray resistance $r_{0}=12.5 \mathrm{~m} \Omega$, the axial mass factor $f_{\mathrm{m}}=0.0013$, the axial current factor $f_{\mathrm{c}}=0.23$, the radial mass factor $f_{\mathrm{mr}}=0.5$, and the radial current factor $f_{\text {cr }}=0.57$, respectively).

It can be observed in Fig. 1 that without the inclusion of anomalous resistivity in the fitting process (the earlier version of Lee's code), the depth of the measured current drop is much more than that of the computed trace. This was noticed in all of the current fitting processes for low-pressure range. It is known that at the end of the compression phase some instabilities and micro instabilities, such as the modified Buneman and low-hybrid [18], occur that lead to the production of anomalous resistances of the order of $1(\Omega)[19,20]$. In plasma focus devices with high static inductance, for the cases where $L_{0} \gg L_{\mathrm{p}}$, these resistances play an important role in the transfer of energy into plasma as well as its consumption by plasma [19]. In these machines, plasma can only consume a small portion of energy stored in magnetic field. This is due to the fact that the value of the plasma inductance is much smaller than the static inductance during the radial phase; therefore, considerable amount of energy is left in the magnetic field. Furthermore, the time period of energy consumption by plasma anomalous resistances, which can be estimated as $\left(L_{0}+L_{\mathrm{p}}\right) / R_{\mathrm{p}}$, is not negligible in high inductance machines. Hence, in these types of devices anomalous resistances can be considered as an alternative to consume the portion of energy that is left in the magnetic field. Lee's computational model is well fitted to machines with low values of static inductance, considerably less than $100 \mathrm{nH}$. For plasma focus devices of higher $L_{0}$, e.g. higher than $100 \mathrm{nH}$, the measured current dip typically has an extended dip, ED, which cannot be fitted by the 5-phase model. A new version of the code has been introduced (RadPFV.6) [11, 20], in which the anomalous resistivity effect has been taken

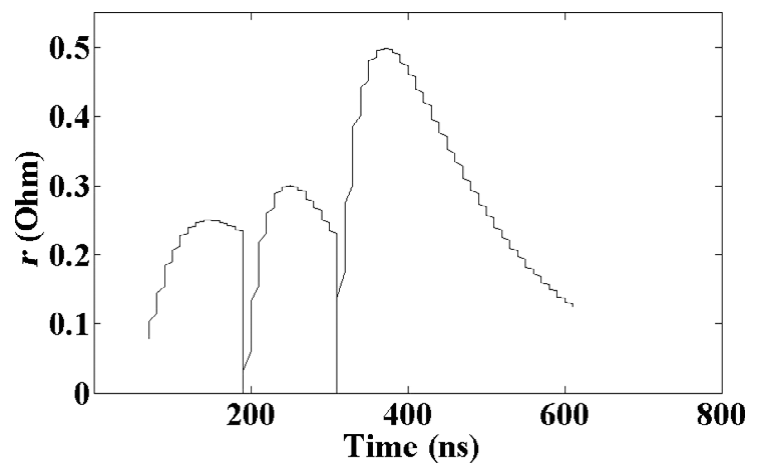

Figure 2. Values of three anomalous resistances from the start of radial phase.

into account. Figure 1 shows that a good agreement between the computed current trace and the measured one can be reached at if the code's new version is implemented. The conditions and parameters of this new fitting process were the same as the ones used for the earlier version except that the new parameters of plasma anomalous resistances were included. Figure 2 shows the amount of anomalous resistances according to the new computed current trace. Three anomalous resistances $\left(R_{1}, R_{2}\right.$, and $\left.R_{3}\right)$ in the form of

$$
R=R_{0}\left[\exp \left(-t / t_{2}\right)-\exp \left(-t / t_{1}\right)\right]
$$

were considered, $R_{0}$ for all three resistances was in the order of $1(\Omega)$, here $t_{1}$ identifies the characteristics of the rise time of the anomalous resistance ( $t_{1}$ was 70 , 65 , and $65 \mathrm{~ns}$ for the three resistances, respectively) and likewise $t_{2}$ represents the characteristics of its fall time ( $t_{2}$ was $150 \mathrm{~ns}$ for all the resistances). In order to prevent these three resistances from mixing up with each other, a parameter called end fraction was considered (the values of this parameter for three resistances, respectively, were $0.7,0.4$, and 1). This parameter acts as a terminator for each anomalous resistance.

To monitor the ion beam emission, the Faraday cup ion detector was used and its measurement sensitivity was kept constant throughout the experiments. Figure 3 shows that increase of the anomalous resistance effect or the current drop at any value of pressure can increase the energy and the flux of the emitted ion beam. An interesting observation in all of the experimental runs, even under low operating pressure regimes, was that as a result of a significant drop in current trace, a dramatic increase in ion beam took place. The appearance of anomalous resistivity leads to an increase in tube voltage, which enhances the acceleration of ions throughout the plasma column. It should be noted that by decreasing the operating pressure, one could increase ion beam energy flux; however, this cannot continue indefinitely because of the following restrictions. First, the maximum reported value of the anomalous resistance for different plasma focus devices is of the order of $1(\Omega)$ [20].

Second, there is a limitation because of the optimum operating pressure of any machine. An alternative way is increasing the plasma focus inductance, which seems 


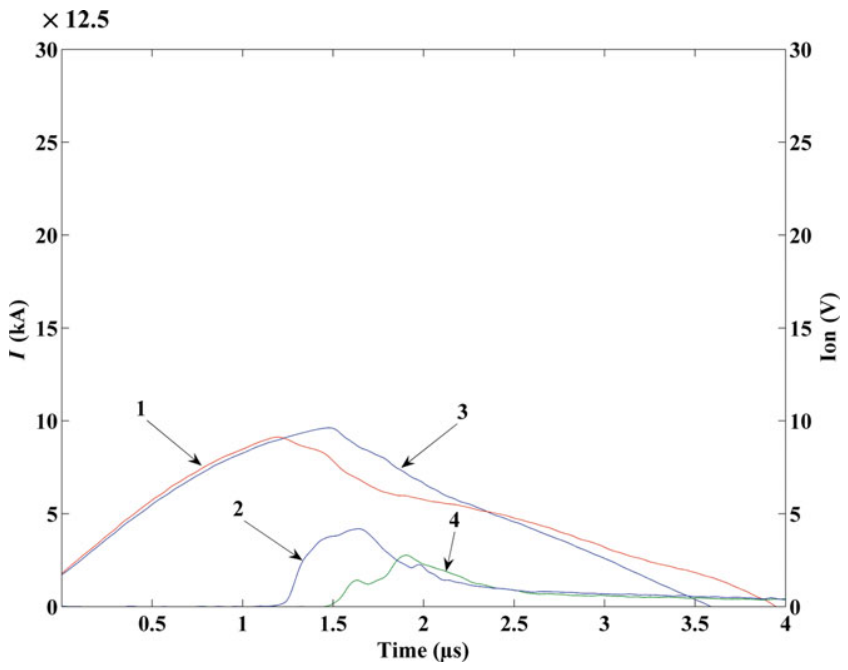

Figure 3. (Colour online) 1-2: measured current and the Faraday cup ion signals at 0.4 mbar. 3-4: same signals at 0.6 mbar. All with $L_{0}=150 \mathrm{nH}$.

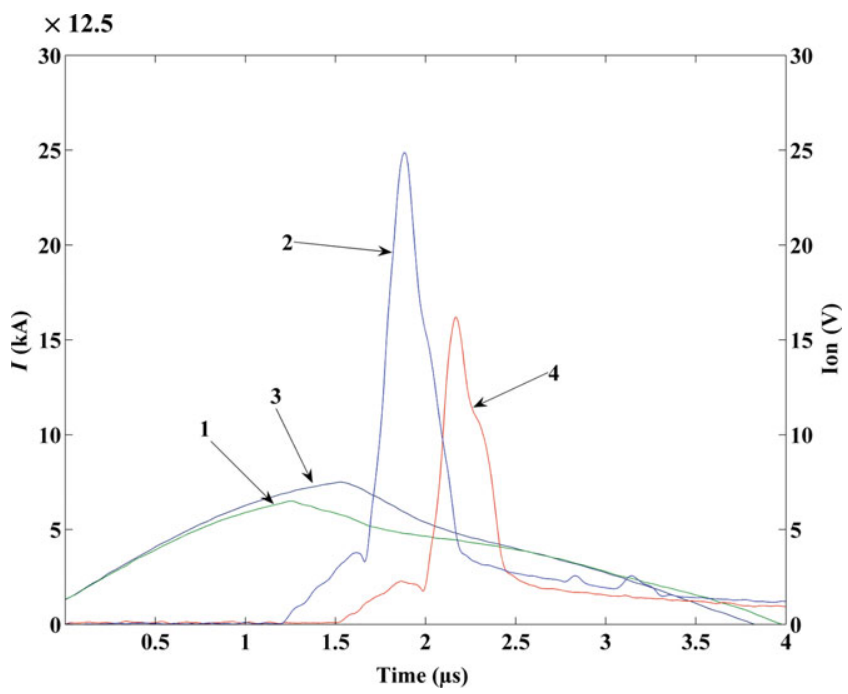

Figure 4. (Colour online) 1-2: measured current and the Faraday cup ion signals at 0.4 mbar. 3-4: same signals at 0.6 mbar. All with $L_{0}=220 \mathrm{nH}$.

to have less limitation than that of the anomalous resistance. In the performed experiments, the anode electrode was directly connected to a single capacitor bank. This configuration provided a minimum total static inductance of the order of $150 \mathrm{nH}$. To study the effects of increased inductance on ion beam emission, the total inductance of the device was gradually increased to $220 \mathrm{nH}$ (the highest operating inductance). This was done through increase of the part of the static inductance that is related to the transmission line of the device. Figure 4 shows that by increasing the static inductance, the energy flux of ion beam emitted from the device has dramatically increased for the same values of pressure as indicated in Fig. 3. It can be argued that by increasing the static inductance or satisfying the condition of $L_{0} \gg L_{\mathrm{p}}$ (which leads to $V_{P F T}=R_{\mathrm{p}} I$ ), the effect of the anomalous resistivity, which plays an important role on ion beam emission before the collapse of plasma column and during the radial phase, has been intensified. Furthermore, increase in static inductance in the plasma focus device can lead to a better condition for the transfer of energy from the capacitance bank (source) to the plasma column (load). This can be achieved through an increase in the inductance of transmission line to a value close to the inductance of the capacitance bank. The total external impedance of the device is of the order of the impedance of the plasma column during the occurrences of anomalous resistances (which is of the order $1 \mathrm{ohm}$ ). It should be noted that by increasing the amount of static inductance, the portion of energy that is left in the magnetic field and can be consumed by anomalous resistances is increased. This fraction of energy is responsible for the main portion of emitted ions in the experiments. To summarize, through increase of static inductance in an appropriate range of pressure, which can drive anomalous resistivity effect, the energy flux of ion beam emitted from the device can increase prominently. Variation in device pressure, which can intensify or lessen the anomalous resistivity effect, is the basic parameter for change in ion beam emission. The highest value of static inductance within the high range of pressure leads to an insignificant production of ions. This is the regime where the anomalous resistivity effect in the experiments is negligible. Following could be the contributory effects: (a) The high inductance has reduced the current too much; and (b) at high pressure the focus comes too late so that the current has not dropped too much.

\section{References}

[1] Bernstein, J. M. 1970 Phys. Fluid 13, 2858.

[2] Gary, S. and Hohl, F. 1973 Phys. Fluid 16, 997.

[3] Imshennik, V. S., Osovets, S. M. and Otroshchenko, T. L. V. 1973 Sov. Phys. JETP 37, 1037.

[4] Kondoh, Y. and Hirano, K. 1978 Phys. Fluid 21, 1617.

[5] Zambreanu, V. and Doloc, C. M. 1992 Plasma Phys. Control. Fusion 34, 1433.

[6] Gary, S. 1974 Phys. Fluid 17, 2135.

[7] Kondoh, Y. 1984 J. Phys. Soc. Jpn. 53, 4200.

[8] Deutsch, R. and Kies, E. 1988 Plasma Phys. Cotrol. Fusion 30, 263.

[9] Zakaullah, M., Omar, A. and Murtaza, G. 1996 Plasma Sources Sci. Technol. 5, 544.

[10] Wong, C. S. and Yap, S. L. 2005 Solid State Phenom. 107, 151.

[11] Lee, S. 2010 Radiative Dense Plasma Focus Computation Package: RADPF. http://www.plasmafocus.net, http://www.plasmafocus.net/IPFS/modelpackage/ File1RADPF.htm, http://www.plasmafocus.net/IPFS/ modelpackage/File2Theory.pdf, http://www.plasma focus.net/IPFS/modelpackage/UPF.htm. Accessed Oct. 19, 2011.

[12] Lee, S. and Saw, S. H. 2008 Appl. Phys. Lett. 92, 021503.

[13] Liu, M. H., Feng, X. P., Springham, S. V. and Lee, S. 1998 IEEE Trans. Plasma. Plasma Sci. 26, 135.

[14] Lee, S., Lee, P., Zhang, G., Feng, X., Gribkov, V. A., Liu, M., Serban, A. and Wong, T. 1998 IEEE Trans. Plasma Sci. 26, 1119. 
[15] Lee, S., Saw, S. H., Lee, P. C. K., Rawat, R. S. and Schmit, H. 2008 Appl. Phys. Lett. 92, 111501.

[16] Gribkov, V. A. et al. 2007 J. Phys. D 40, 3592.

[17] Lee, S. 2009 Appl. Phys. Lett. 95, 151503.

[18] Davidson, R. C. and Krall, N. A. 1977 Nucl. Fusion 17, 6.
[19] Bernard, A. et al. 1998 Moscow J. Phys. Soc. 8, 93.

[20] Lee, S., Saw, S. H., Abdou, A. E. and Torreblanca, H. 2011 Characterizing plasma focus devices-role of the static inductance-instability phase fitted by anomalous resistances. J. Fusion. Energ. 30, 4. 